\title{
The spatial stability of a class of similarity solutions
}

\author{
L. Durlofsky and J. F. Brady \\ Department of Chemical Engineering, Massachusetts Institute of Technology, Cambridge, Massachusetts \\ 02139
}

(Received 31 May 1983; accepted 4 November 1983)

\begin{abstract}
The spatial stability of a class of exact similarity solutions of the Navier-Stokes equations whose longitudinal velocity is of the form $x f^{\prime}(y)$, where $x$ is the streamwise coordinate and $f^{\prime}(y)$ is a function of the transverse, cross-streamwise, coordinate $y$ only, is determined. These similarity solutions correspond to the flow in an infinitely long channel or tube whose surface is either uniformly porous or moves with a velocity linear in $x$. Small perturbations to the streamwise velocity of the form $x^{\lambda} g^{\prime}(y)$ are assumed, resulting in an eigenvalue problem for $\lambda$ which is solved numerically. For the porous wall problem, it is shown that similarity solutions in which $f^{\prime}(y)$ is a monotonic function of $y$ are spatially stable, while those that are not monotonic are spatially unstable. For the accelerating-wall problem, the interpretation of the stability results is not unambiguous and two interpretations are offered. In one interpretation the conclusions are the same as for the porous problem-monotonic solutions are stable; the second interpretation is more restrictive in that some of the monotonic as well as the nonmonotonic solutions are unstable.
\end{abstract}

\section{INTRODUCTION}

Similarity solutions to the equations of motion have been in use at least since Blasius ${ }^{1}$ assumed a self-similar velocity profile for the boundary-layer flow over a flat plate. Many of the exact solutions to the Navier-Stokes equations are based on the assumption of self-similarity; thus reducing the equations of motion to one or more ordinary differential equations and greatly simplifying the analysis. Important as similarity solutions are in helping us understand the behavior of fluids, there is no assurance that these solutions represent a physically realizable flow. The flow domains are often unbounded, and the similarity solutions possess singularities either at infinity or at the origin; thus casting some doubt as to their uniform validity. It has generally been accepted, however, that similarity solutions can and do provide at least a local description of some flow. Several recent studies have examined the question of the applicability of similarity solutions and have shown that even a local validity may not be possible. $^{2-6}$

In this paper we wish to investigate the validity of a particular class of similarity solutions. These solutions correspond to the flow in a uniformly porous channel or tube and to the flow in a channel or tube with an accelerating surface velocity (cf. Fig. 1). The similarity solutions for the porous wall problem have been studied by Terrill and others, ${ }^{7-12}$ and those for the accelerating surface problem by Brady and Acrivos. ${ }^{13} \mathrm{We}$ are interested in these problems because the similarity solutions possess some rather unusual, and perhaps unphysical, features such as the existence of multiple solutions for some range of Reynolds number. Even more disturbing, however, is the discrepancy which exists between the axisymmetric (tube) and two-dimensional (channel) flows for positive Reynolds numbers for both the porous wall and accelerating surface problems: a range of Reynolds number exists within which there is no solution to the axisymmetric similarity equation, while solutions to the two-dimensional case exist for all values of the Reynolds number. This inconsistency and lack of similarity solutions for the axisymmetric problems calls into question their validity.

Brady and Acrivos ${ }^{5}$ addressed this paradox of nonexistence in their study of the flow in a channel or tube with an accelerating surface velocity. Its cause was traced to the assumption that the tube and channel were infinite in extent. By an appropriate, boundary-layer-like, numerical analysis of the equations of motion, they showed that above a critical value of the Reynolds number $\mathbf{R}_{c}$, the flow throughout the entire domain was influenced by the end condition. Thus, the similarity solution no longer represented any real flow. Recently, Brady ${ }^{6}$ has shown that the same phenomenon occurs in the porous wall problem. It should be noted, however, that although the domain is finite, the actual length of the channel or tube can be arbitrarily large.

The end condition may be viewed as an $O(1)$, mass-conserving, numerical perturbation to the similarity velocity profile. At low values of $R$, this perturbation decayed as it was convected longitudinally away from the end, and the similarity solution was valid over a region far removed from the end. At $\mathbf{R}_{c}$, however, the perturbation was convected all the way to the origin, and the region of validity of the similarity solution shrunk to zero. Further, Brady and Acrivos ${ }^{5}$ found that this critical value of $\mathbf{R}$ was insensitive to the detailed nature of the end condition. For flow in a tube with an accelerating surface velocity, $\mathbf{R}_{c}$ coincided exactly with the value of $R, 10.25$, beyond which no solution to the similarity equation existed. A critical $\mathbf{R}$ was also shown to exist for flow in a channel with an accelerating surface velocity $\left(R_{c} \cong 57\right)$, even though solutions to the similarity equation exist for all $\mathbf{R}$. For the porous wall problem (Ref. 5), critical Reynolds numbers of 2.3 and 6, respectively, were also found. It was shown, however, that the similarity solutions for the porous wall problem may regain validity at larger $\mathbf{R}$ if the end condition is sufficiently "close" to a similarity solution. Thus, we see that beyond $\mathbf{R}_{c}$ the similarity solutions for this class of flows may or may not describe the motion in a 
finite channel or tube.

The important influence of the end condition and the presence of critical Reynolds numbers suggests that a spatial stability analysis of the similarity equations may aid in understanding how and why these solutions lose validity. In particular, it would be of interest to see if these critical Reynolds numbers could be predicted by such an analysis. Because these similarity equations are very much like the classical boundary-layer equations, it is instructive to first consider the spatial stability of the Falkner-Skan equation for the boundary-layer flow over a wedge. Serrin ${ }^{14}$ proved that for an arbitrary initial velocity profile, the magnitude of the deviation of the actual flow from that predicted by the similarity solution is $o\left[(1+m \log x) / x^{m}\right]$ for $m \geqslant 0$ as $x \rightarrow \infty$ downstream. [Here, $m$ is the exponent of the free stream velocity $U(x)=C x^{m}$, where $C$ is a constant. His analysis is restricted to the case $m \geqslant 0$.] Thus, for these flows an $O(1)$ perturbation to the similarity solution, regardless of its form, will decay as it is convected downstream; i.e., the similarity solution will be valid asymptotically far downstream from the leading edge.

Chen and Libby ${ }^{15}$ also investigated the validity of solutions to the Falkner-Skan equation by means of a stability analysis. They assumed small perturbations to the streamwise velocity of the form $x^{\lambda} \phi(\eta)$, where $x$ is the distance from the leading edge, $\eta$ is the similarity variable, and $\phi(\eta)$ is the perturbation function. Here $\lambda$ is the unknown eigenvalue: $\lambda<0$ represents stability in the sense that small perturbations will decay as $x \rightarrow \infty$ (downstream), and $\lambda>0$ would therefore indicate spatial instability. ${ }^{16}$ For $m \geqslant 0$, only negative eigenvalues were found, in agreement with Serrin's analysis. For $m<0$, the solutions to the Falkner-Skan equation are no longer unique, there being at least two different solutions. One branch of solutions has reverse flow adjacent to the surface-fluid moving towards the leading edge, and it connects with the unidirectional flow branch at the point where the shear stress at the surface vanishes, $m=-0.0904$. For the reverse flow branch, they found both positive and negative $\lambda$ 's and attributed the presence of the positive eigenvalues as an indication of instability (see Sec. II). The undirectional flow branch gave only negative $\lambda$ 's. The agreement between the analyses of Chen and Libby ${ }^{15}$ and Serrin ${ }^{16}$ for $m \geqslant 0$ and the conclusions concerning the stability of the branches for $m<0$ indicate the utility of a linear stability analysis for determining solution validity.

Our approach will be quite similar to that of Chen and Libby. ${ }^{15}$ In Sec. II we consider the spatial stability of the two-dimensional porous wall and accelerating surface problems. We show that the stability results may be interpreted in two ways. One interpretation applies to both the porous and accelerating surface problems and maintains that the similarity solutions which are monotonic functions of the crossstreamwise coordinate are stable, while those that are not are unstable. The other interpretation applies only to the accelerating surface problem and further limits the spatially stable flows. It implies that all of the nonmonotonic solutions are unstable and that the monotonic solutions, which exist for $-\infty<R<\infty$, are stable only for $0<R<11.0$. In neither case, then, are we able to determine the critical Reyn- olds numbers reported in Refs. 5 and 6. Section III is devoted to the axisymmetric tube problems. Here, a critical Reynolds number is found, and it agrees precisely with the Reynolds number at which similarity solutions cease to exist. The conclusions we reach regarding stability or instability of the similarity solutions are with regard to a particular class of spatial perturbations. Questions of temporal stability and stability to a broader class of spatial perturbations are not addressed in this study.

\section{THE TWO-DIMENSIONAL CHANNEL PROBLEMS}

\section{A. Porous channel flow}

Referring to Fig. 1, we denote the channel half-width by $h$, the fluid viscosity by $\mu$, its density by $\rho$, and the normal velocity through the porous wall by $V$. For an infinitely long channel, the Navier-Stokes equations admit an exact similarity solution of the form

$$
\begin{aligned}
& u=-x f^{\prime}(y), \\
& v=f(y), \\
& p=p_{0}(y)+\frac{1}{2} \beta x^{2},
\end{aligned}
$$

where $u$ and $v$ are the $x$ and $y$ components of velocity, $p$ is the pressure, and $\beta$ is a constant. For flows symmetric about $y=0$, the similarity function $f$ satisfies

$$
\begin{aligned}
& f^{\prime \prime \prime}+\beta=-\mathrm{R}\left[\left(f^{\prime}\right)^{2}-f f^{\prime \prime}\right], \\
& f(0)=f^{\prime \prime}(0)=0, \quad f(1)=1, \quad f^{\prime}(1)=0 .
\end{aligned}
$$

Here, $\mathbf{R}=\rho V h / \mu$ is the Reynolds number; $\mathbf{R}>0$ corresponds to suction, and $\mathbf{R}<0$ to injection. The unknown pressure coefficient $\beta$ is determined by the fourth boundary condition.

The solutions to Eqs. (1) and (2) have been studied extensively, ${ }^{7-11}$ and we shall comment only briefly on their structure. There exists a single continuous solution extending from $-\infty$ to $+\infty$ in $\mathbf{R}$. The longitudinal velocity profiles are monotonic functions of $y$, with the fluid coming in from infinity for suction $(R>0)$ and going out towards infinity for injection $(R<0)$. The suction solutions develop a boundary layer near the wall as $R \rightarrow \infty$; the injection solutions do not. There are two additional suction solutions which appear at $R=12.165$ and extend to $R \rightarrow+\infty$. These solutions have velocity profiles in which the longitudinal velocity is no longer a monotonic function of $y$, and for some solutions there is a region of reverse flow at the centerline with the fluid there now moving out towards infinity. In Fig. 2 we show a plot of $\beta$ vs $\mathbf{R}$ where we have labeled the different

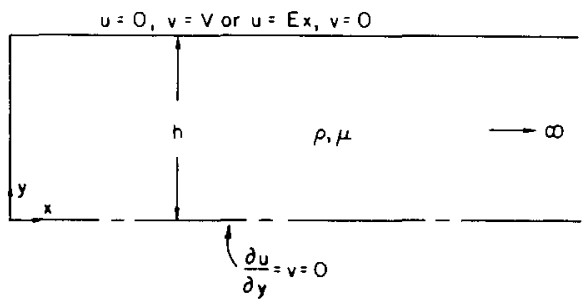

FIG. 1. Schematic diagram for the two-dimensional channel similarity solutions. Uniformly porous: $u=0, v=V$. Accelerating surface: $u=E x$, $v=\mathbf{0}$. 


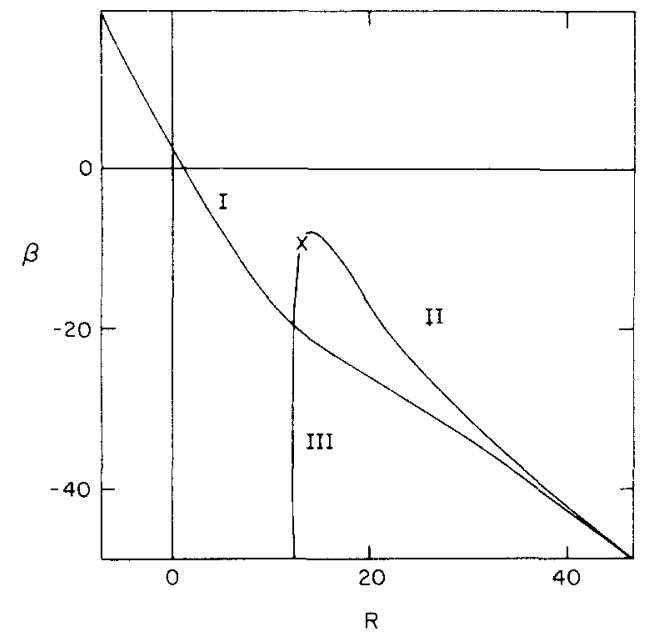

FIG. 2. The pressure coefficient $\beta$ of the similarity solutions for the twodimensional flow in a uniformly porous channel as a function of the Reynolds number. The three solution families are labeled I, II, and III.

solutions I, II, and III. The point X marks the juncture of branches II and III and is the point where the centerline velocity is zero.

To determine the spatial stability of these similarity solutions, we write for the velocity and pressure fields

$$
\begin{aligned}
& u=-x f^{\prime}(y)-x^{\lambda} g^{\prime}(y), \\
& v=f(y)+\lambda x^{\lambda-1} g(y), \\
& p=p_{0}(y)+\frac{1}{2} \beta x^{2}+[1 /(1+\lambda)] \beta_{\lambda} x^{1+\lambda,}
\end{aligned}
$$

where $g^{\prime}$ is the perturbation to the longitudinal velocity and $\beta_{\lambda}$ is the perturbation to the pressure. Substituting into the $x$-momentum equation and neglecting terms of second order in the small perturbation $g$, the linearized stability equation for $g$ is

$$
g^{\prime \prime \prime}+\beta_{\lambda}=\mathbf{R}\left[f g^{\prime \prime}-(1+\lambda) f^{\prime} g^{\prime}+\lambda f^{\prime \prime} g\right],
$$

with

$$
g(0)=g^{\prime \prime}(0)=g(1)=g^{\prime}(1)=0, \quad g^{\prime}(0)=1,
$$

where without loss of generality we have set $g^{\prime}(0)$ equal to unity. Both $\lambda$ and $\beta_{\lambda}$ are unknown eigenvalues. Given a similarity solution $f$ at a particular $\mathbf{R}$, the two-point boundaryvalue problem [(4) and (5)] was solved using Newton's method to determine $g, \beta_{\lambda}$, and $\lambda .{ }^{17}$

Whether the eigenvalue $\lambda$ is greater than or less than unity will determine the spatial stability of the similarity solutions. Some care is needed, however, when considering in which direction, increasing or decreasing $x$, the perturbations propagate. For the group I solutions, a negative Reynolds number corresponds to injection with the fluid moving out towards infinity. Here, we wish to consider a perturbation occurring at some finite value of $x$ and ask whether this perturbation decays relative to the similarity solution as it is convected with the flow as $x \rightarrow \infty$. Thus, $\lambda<1$ represents stability. For positive Reynolds numbers corresponding to suction, the situation is reversed. The flow is now moving inward from infinity to the origin $x=0$. Here, we imagine a perturbation at some finite $x$ and ask whether it decays relative to the similarity solution as $x \rightarrow 0 .{ }^{18}$ Thus, $\lambda>1$ repre- sents stability. For the group III solutions in which there is fluid moving in both the positive and negative $x$ directions, the direction of propagation of the perturbation and hence the stability criterion with $\lambda$ are no longer as unambiguous. This difficulty becomes even more apparent in the accelerating surface problems because fluid always moves in both directions. When we consider the accelerating-surface channel problem later in this section, we shall offer an interpretation of $\lambda$ with regard to the direction of flow. For the moment, we shall simply consider the group I porous channel results.

The minimum magnitude eigenvalues $\lambda$ for the group I porous channel solutions are plotted versus the Reynolds number in Fig. 3. No negative $\lambda$ 's were found for $R>0$ (suction), nor were there positive $\lambda$ 's for $\mathbf{R}<0$ (injection). For $\mathbf{R}>0, \lambda$ asymptotically approaches 1 as $\mathbf{R} \rightarrow \infty$; thus, suction flow is always stable, approaching marginal stability as $\mathbf{R}$ tends to infinity. For $\mathbf{R}<0, \lambda$ asymptotically approaches -3 as $\mathbf{R} \rightarrow-\infty$; injection flow is always stable, even asymptotically in $\mathbf{R}$. The asymmetry in the asymptotic forms of $\lambda$ vs $\mathbf{R}$ is not surprising because the similarity flows are themselves quite different at large $|\mathbf{R}|:$ a boundary layer adjacent to the wall forms in suction flow, but not for injection. The similarity solutions are, however, symmetric about $\mathbf{R}=0$, and this symmetry is present in the stability analysis. As $R \rightarrow 0$, from above or below, $\lambda \sim \lambda_{0} / \mathrm{R}$ with $\lambda_{0}=18.81$. There are of course additional eignevalues, and they show the same behavior as the minimum magnitude eigenvalues, with different $\lambda_{0}$ 's and asymptotic values for large $|\mathbf{R}|$.

The spatial stability analysis of the group I solutions has shown these solutions to be stable for all R. We have not found (as was initially hoped) a critical Reynolds number for loss of stability. The predictions of the stability analysis for $\mathbf{R}>0$ do seem, however, to agree well with the numerical results of Ref. 6 . There it was shown that for $R \gtrsim 50$, certain $O(1)$ numerical perturbations to the inlet condition result in velocity and pressure profiles as $x \rightarrow 0$ that are superpositions

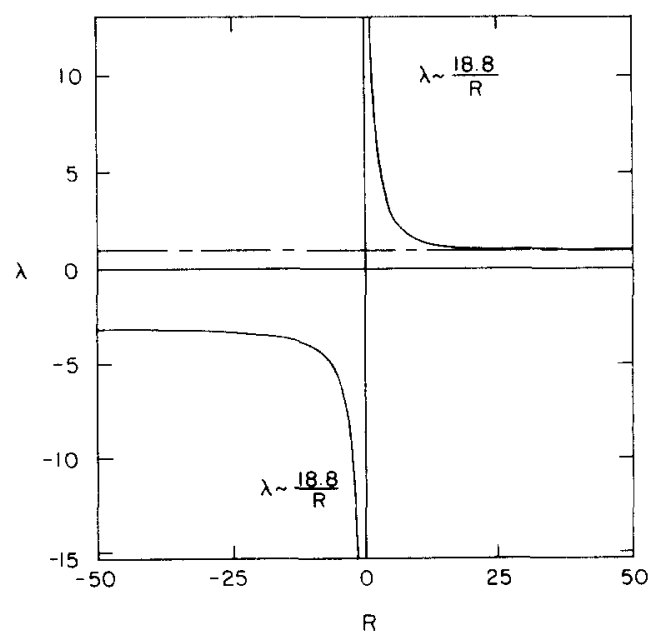

FIG. 3. The minimum magnitude eigenvalues $\lambda$ for the group 1 porous channel solutions. Here $\lambda \sim 1$ as $\mathbf{R} \rightarrow \infty, \lambda \sim-3$ as $\mathbf{R} \rightarrow-\infty$, and $\lambda \sim 18.81 / R$ as $R \rightarrow 0$. The eigenvalues indicate the group $I$ solutions are spatially stable. The dashed lines in this and all subsequent figures of stability results correspond to $\lambda=1$. 
of the similarity solution and perturbations, $g^{\prime}(y)$ and $\beta_{\lambda}$, with $\lambda=1$ in excellent agreement with the stability analysis.

\section{B. Accelerating channel flow}

The accelerating-wall channel flow is described by the same similarity form- $u=-x f^{\prime}(y)$, etc. - as the porous channel flow. The only differences are that the boundary conditions at the surface are $u=E x$ and $v=0$ in lieu of $u=0, v=V$, giving $f^{\prime}(1)=-1, f(1)=0$ in Eq. (2), and the Reynolds number is $\mathrm{R}=\rho E h^{2} / \mu$. The solution structure is analogous to the porous channel case, resulting in a $\beta$ vs $\mathbf{R}$ plot of the same form as shown in Fig. 2. There are again three different solutions for $\mathbf{R}>0$, groups I, II, and III, with the Reynolds number at which multiple solutions appear now being 306. As in the porous channel case, the group I solutions are monotonic functions of $y$ and extend from $-\infty$ to $+\infty$ in $\mathbf{R}$, while the groups II and III are not monotonic functions of $y$. For a detailed discussion of these similarity solutions for $\mathbf{R}>0$, see Ref. 13 .

One aspect of the accelerating wall problem that is different from the porous wall problem is the presence of fluid moving in both directions. For $\mathrm{R}<0$, a decelerating surface, fluid is carried inward from infinity along the moving surface and returns to infinity on the centerline. For the group $I$ solutions with $\mathbf{R}>0$, the situation is the opposite, with fluid moving out towards infinity along the moving surface and returning on the centerline. Thus, for all Reynolds numbers there is reverse flow, and the direction of propagation of a disturbance and the interpretation of stability require some care. Proceeding as before with the stability analysis, the minimum magnitude eigenvalues $\lambda$ for the group I solutions are shown in Fig. 4.

In Fig. 4 we see that, unlike the porous channel problem, there are both positive and negative eigenvalues for $R>11.0$, only positive eigenvalues for $0<R<11.0$ and only negative eigenvalues for $R<0$. A limit point exists at

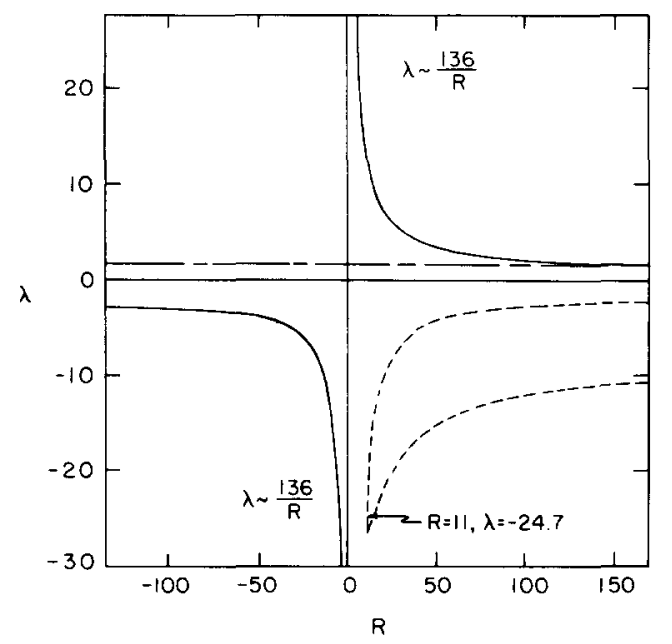

FIG. 4. The minimum magnitude eigenvalues $\lambda$ for the group I accelerating channel solutions. Under the second interpretation, the solid curves correspond to fluid moving towards infinity for $R<0$, and towards $x=0$ for $\mathbf{R}>0$. Here, $\lambda \sim 1$ as $\mathbf{R} \rightarrow \infty, \lambda \sim-2$ as $\mathbf{R} \rightarrow-\infty$, and $\lambda \sim 136 / \mathbf{R}$ as $\mathbf{R} \rightarrow 0$. The dashed curves correspond to the fluid moving towards infinity in the boundary layer adjacent to the accelerating wall. Here, $\lambda \sim-2$ and -8.20 as $R \rightarrow \infty$. There is also a limit point at $R=11.0$ with $\lambda=-24.7$.
$\mathbf{R}=11.0$ with $\lambda=-24.7$. (There are of course additional branches of negative eigenvalues lying below the dashed curves in Fig. 4. These branches have the same form as those presented, but the limit points are all at larger R.) As $\mathbf{R} \rightarrow+\infty$, the positive eigenvalues asymptotically approach 1 , as do their porous channel counterparts, and the maximum negative eigenvalues approach -2 . The significance of the positive and negative eigenvalues is a matter of some debate in that there are different ways of interpreting these results. We shall offer two interpretations which we feel are plausible and point out both their strengths and weaknesses. It is hoped that future work in this area will help resolve this issue.

The first interpretation proceeds as follows. Because there is always fluid moving towards $x=0$, the presence of negative eigenvalues for $R>11$ implies instability. While this interpretation seems quite straightforward and predicts the existence of a critical Reynolds number, there are, however, some disturbing features. If the negative eigenvalues imply instability for the fluid moving towards $x=0$, then the positive eigenvalues should imply instability for the fluid moving toward $x \rightarrow \infty$. Thus the flow is unstable for all $\mathbf{R}$, becoming increasingly so as $\mathbf{R} \rightarrow 0$, where $\lambda \rightarrow \infty$. It may be argued, however, that $x$ never really reaches $\infty$, and that the end of the channel will occur for $x \sim O(L)$, where $L$ is some large $(L / h>1)$ but finite length. Thus, if the amplitudes of the $\lambda>0$ perturbations are sufficiently small, they will be convected out of the channel before they can grow to render the similarity solution invalid.

While it is arguable that the finite length $L$ plays a role in interpreting the positive $\lambda$ 's, it should be realized that there are infinitely many positive $\lambda$ branches all giving $\lambda \rightarrow \infty$ as $\mathbf{R} \rightarrow 0$, and thus the amplitudes for all these branches must be small. Furthermore, this interpretation also implies that the decelerating channel flow $(\mathrm{R}<0)$ is unstable for all negative $\mathbf{R}$, becoming "most unstable" as $\mathbf{R} \rightarrow 0^{-}$. For the decelerating channel there are only negative $\lambda$ 's, and fluid is always moving towards $x=0$. We have, therefore, the rather curious situation where the flow as $R \rightarrow 0^{-}$is unstable while that for $\mathbf{R} \rightarrow 0^{+}$is stable; this is surprising because the similarity function $f$ is symmetric about $\mathrm{R}=0$ and, more importantly, because one does not normally expect instability as $|\mathbf{R}| \rightarrow 0$.

The second interpretation of the eigenvalues shown in Fig. 4 arises from a consideration of the flow as $R \rightarrow \infty$. At large $R$, the flow consists of two distinct regions moving in opposite directions: an $O\left(\mathrm{R}^{-1 / 2}\right)$ thin boundary layer adjacent to the surface moving towards increasing $x$ and an inviscid, uniform, zero-vorticity core of stregnth $O\left(\mathrm{R}^{-1 / 2}\right)$ moving towards $x=0$. This interpretation contends that the negative eigenvalues correspond to the flow in the boundary layer (fluid moving towards $+\infty$ ) while the positive eigenvalues correspond to the flow in the core (fluid moving towards $x=0$ ). Under this interpretation, the flow is stable for all $R$, with the flow in the core becoming marginally stable $(\lambda \rightarrow 1)$ as $R \rightarrow \infty$. Negative eigenvalues do not exist for $R<11.0$ because, at small $R$, a well-formed boundary layer does not exist and the flow cannot be separated into two distinct regions. 
The contention that the negative eigenvalues apply to the boundary layer is consistent with several other results. First, we performed a spatial stability analysis of the boundary layer equations resulting from an asymptotic analysis of the similarity solution as $\mathrm{R} \rightarrow \infty$. (This boundary-layer problem is the moving wall equivalent of the Falkner-Skan equation with $m=1$; that is, it is to the Falkner-Skan equation as the Sakiadis ${ }^{19.20}$ moving wall boundary layer is to the Blasius equation.) The maximum eigenvalue for this problem is -2 , exactly as is observed for the similarity solution; the next lower eigenvalue is -8.20 , in agreement with the asymptote of the lower curve in Fig. 4. Therefore, at least as $\mathrm{R} \rightarrow \infty$, the negative eigenvalues do seem to correspond to the boundary layer.

Second, for the decelerating surface case, $R<0$, only negative eigenvalues exist. Here, the flow does not form a boundary layer as $R \rightarrow-\infty$; two distinct regions of flow do not exist; and, therefore, two branches of eigenvalues are not required. Furthermore, the similarity solution is symmetric about $\mathbf{R}=0$ which is reflected in the solid curves in Fig. 4, where $\lambda \sim \lambda_{0} / R$ as $R \rightarrow 0$ from above and below with $\lambda_{0}=136$. As $R$ passes through zero, the flow changes direction, and, as the positive eigenvalues represent stability for $\mathbf{R}>0$, the negative eigenvalues represent stability for $\mathbf{R}<0$. Thus, this interpretation also implies that the decelerating surface flow is stable, even asymptotically as $R \rightarrow-\infty$ where $\lambda \rightarrow-2$.

Finally, this interpretation is able to explain the difference in the stability results for the porous and accelerating channel flows. In both problems the group I solutions are stable for all $\mathbf{R}$, becoming marginally stable as $\mathbf{R} \rightarrow \infty$. In fact, the solid curves in Fig. 4 look much like those in Fig. 3. It is only the negative eigenvalues for $R>11.0$ that differ between the two problems, and these negative eigenvalues have been attributed to the boundary layer flow which moves toward $x \rightarrow \infty$, in the direction opposite to that of the core flow. Because such a boundary layer does not exist in the group I porous channel flow (the boundary layer moves in the same direction as the core, towards $x=0$ ), no negative eigenvalues would be expected to exist.

Although this interpretation is able to consistently explain the stability results for positive and negative $R$, it rests on the premise that the flow can be separated into two regions-a boundary layer and a core. This allows the negative eigenvalues to be associated with the boundary layer and the positive eigenvalues with the core. While firmly established as $R \rightarrow \infty$, it is not certain that this correspondence continues to apply as $\mathrm{R}$ decreases from infinity.

\section{Porous and accelerating channel flow: Groups II and III}

We shall now show that the groups II and III solutions for both the porous and accelerating channel flows are unstable. Referring to Fig. 2 for the porous channel flow, we should note that the group II solutions asymptotically approach the group I solutions as $\mathbf{R} \rightarrow \infty$. As $\mathbf{R}$ decreases along the group II branch, the longitudinal velocity $u$ is no longer monotonic, and the minimum in $u$ moves off the centerline until the point $X$ at $R=13.1$ is reached where the centerline velocity is zero. As we continue along the group III branch (at first decreasing and then increasing in R), the centerline velocity becomes positive and increases in magnitude. A similar evolution in structure occurs for the accelerating channel groups II and III solutions.

The stability results for the porous and accelerating channel solutions are shown in Figs. 5 and 6, respectively, with the curves labeled according to the group to which they belong. For the porous channel group II solutions only positive eigenvalues were found. The lowest branch, however, is always less than unity, approaching 1 asymptotically from below as $\mathbf{R} \rightarrow \infty$ where the groups I and II solutions join. Thus, the group II solutions are spatially unstable. The results are the same for the accelerating channel group II solutions, except that there is a set of negative eigenvalues. This negative branch asymptotically approaches $\lambda=-2$ as $\mathbf{R} \rightarrow \infty$ where it joins the group I negative eigenvalues. Again, the accelerating channel stability results may be interpreted in two ways, but in this case the two interpretations yield the same conclusion. Under the first interpretation the flow is unstable due to the existence of eigenvalues $\lambda$ less than unity (both positive and negative $\lambda$ 's). Under the second interpretation, the negative eigenvalues do not imply instability; they correspond to the boundary layer and indicate that it is stable. However, the positive $\lambda$ 's which are less than 1 apply to the flow in the core and indicate instability. Thus the group II solutions for both problems are spatially unstable.

For both group III solutions, there is a small region in Reynolds number where there are positive $\lambda$ 's, with $\lambda$ passing through 1 at the minimum Reynolds number. There is a branch of negative $\lambda$ 's which asymptotically approaches $\lambda=0$ as $\mathrm{R} \rightarrow \infty$ and either connects with the group II solutions in the accelerating channel problem, or returns to $\mathbf{R} \rightarrow \infty$ in the porous channel problem. Under the first interpretation, group III solutions for both flows are unstable because negative eigenvalues exist for all possible values of $R$. In the second interpretation, since the group II negative

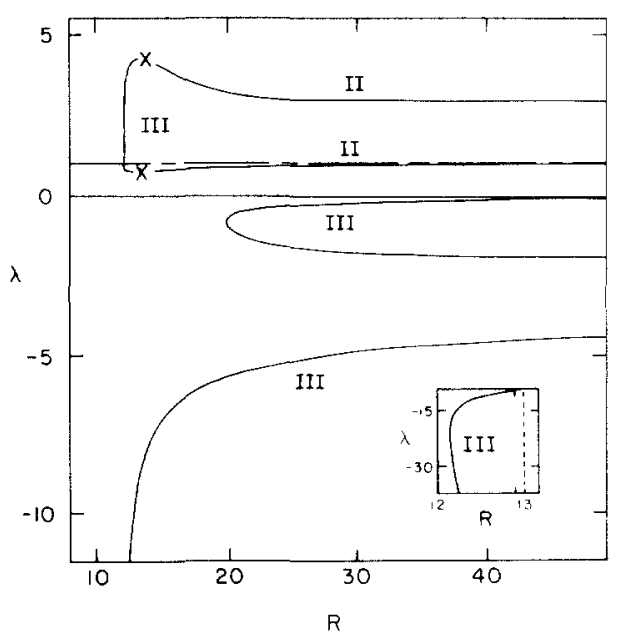

FIG. 5. The minimum magnitude eigenvalues $\lambda$ for the groups II and III porous channel solutions. The inset shows that the group III negative $\lambda$ curve is multivalued because there are two group III solutions in the range $12.165 \leqslant R \leqslant 13.119$ (cf. Fig. 2). The solutions are unstable. 


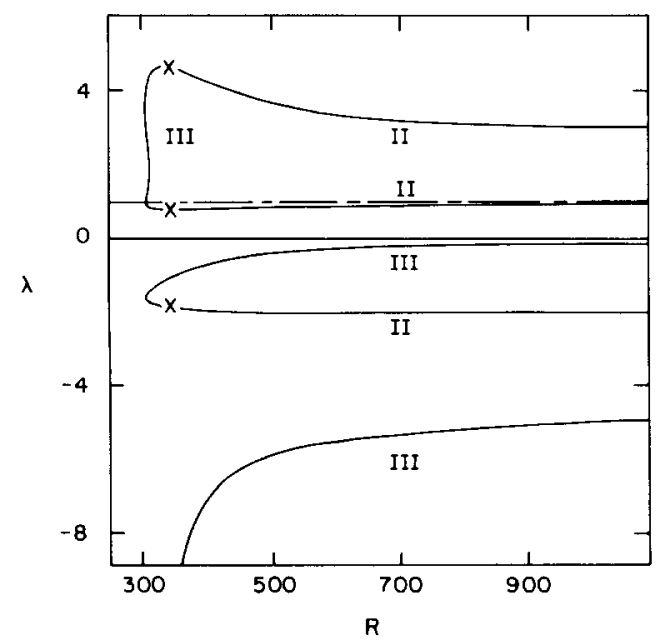

FIG. 6. The minimum magnitude eigenvalues $\lambda$ for the groups II and III accelerating channel solutions. The inset of Fig. 5, although present, is not shown. The solutions are unstable.

eigenvalues for the accelerating channel flow are associated with the boundary layer, the connecting group III branch must also be associated with the boundary layer. The presence of negative, boundary-layer, $\lambda$ 's for the group III porous channel problem, when there are none for the group II is not surprising because the structure of the boundary layers for these two groups is different (see Ref. 13). Since the boundary-layer flow is moving towards the origin, these negative $\lambda$ 's represent an unstable boundary layer. Finally, both group III solutions have a branch of very negative eigenvalues that starts with $\lambda \rightarrow \infty$ at the point $\mathrm{X}$ where the centerline velocity first becomes positive. Chen and Libby ${ }^{15}$ found the same behavior when they investigated the stability of the Falkner-Skan equation. When $m<0$ they found the stability analysis of the reverse flow solution to have negative $\lambda$ 's with $\lambda \rightarrow-\infty$ as $m \rightarrow-0.0904$, the point of zero shear stress where the velocity at the wall first reverses. Chen and Libby concluded that the presence of these negative eigenvalues represents spatial instability. We agree with their conclusion, and interpret our group III negative $\lambda$ 's as representing instability of the reversed flow on the centerline.

The spatial stability analysis of the porous and accelerating channel flows has led to the following conclusions: (1) group I porous channel flows are stable; (2) group I accelerating or decelerating channel flows are, depending on which interpretation is applied, either (a) stable for $0<R<11.0$ and unstable for all other $\mathbf{R}$ or (b) stable for all $\mathbf{R}(-\infty<\mathbf{R}<\infty)$; and, (3) groups II and III solutions for both flows are unstable. The key feature that seems to distinguish the groups II and III solutions is the fact that the longitudinal velocity profiles are not monotonic functions of $y$. Thus, we see that the nonmonotonic solutions are unstable, while the monotonic solutions are either stable for all $\mathbf{R}$ or stable over a limited range of $R$ for the accelerating channel flow if the first interpretation is followed. In the next section, we shall see that monotonicity also plays a central role in the axisymmetric tube problems.

\section{THE AXISYMMETIC TUBE PROBLEMS}

The analysis of the axisymmetric tube flow follows along the same lines as the channel flow considered in Sec.
II. Here, the similarity transformation for the axial velocity is $u=-x f^{\prime}(r) / r$; for the radial velocity, $v=f(r) / r$, and for the pressure $p=p_{0}(r)+\frac{1}{2} \beta x^{2}$. The Reynolds number is unchanged, with $\mathbf{R}>0$ being suction or accelerating and $\mathbf{R}<0$ injection or decelerating. Since the porous and accelerating tube problems are quite similar, only the porous problem will be discussed in any detail. The solution structure for the porous problem is shown in Fig. 7 where $\beta$ is plotted versus the Reynolds number. The various solution families have been labeled I, II, IV(i), IV(ii), V(i), and V(ii) to be consistent with the classification scheme of Terrill and Thomas ${ }^{9}$ and Skalak and Wang. ${ }^{11}$

The group I axial velocity profiles are monotonic functions of $r$ and evolve continuously from $R \rightarrow-\infty$ to $\mathbf{R}=2.3$ at which point the shear stress at the wall, $f^{\prime \prime}(1)$, vanishes. These solutions are analogous to the porous channel group I solutions. The group II joins with the group I at $R=2.3$ and evolves continuously as $R$ decreases to zero. These axial velocity profiles are not monotonic functions of $r$, having a single inflection point and a region of reverse flow-fluid moving out towards infinity-adjacent to the wall. The group IV(i) solutions exist in the range $20.6 \leqslant R<\infty$ and are characterized by a region of reverse flow between the tube wall and centerline. Group IV(ii) solutions exist for $23.7 \leqslant R<\infty$, joining with the group IV(i) at $R=23.7$, the point labeled $X$ in Fig. 6 . These solutions do not have reverse flow, but their axial velocity profiles are not monotonic, having two inflection points. Neither groups II nor IV have a corresponding branch in the channel flow. Group V(i) solutions exist for $9.99 \leqslant R<\infty$ and have a region of reverse flow on the centerline. They join with the group $V$ (ii) solutions at the point $X, R=9.99$, where the centerline velocity is zero. The group $\mathrm{V}$ (ii) solutions do not have a region of reverse flow, but do have a velocity profile with an inflection point. The groups $V(i i)$ and $V(i)$ correspond to the porous channel groups II and III, respectively. As $\mathbf{R} \rightarrow \infty$, the groups IV(ii) and V(ii) asymptotically tend toward the

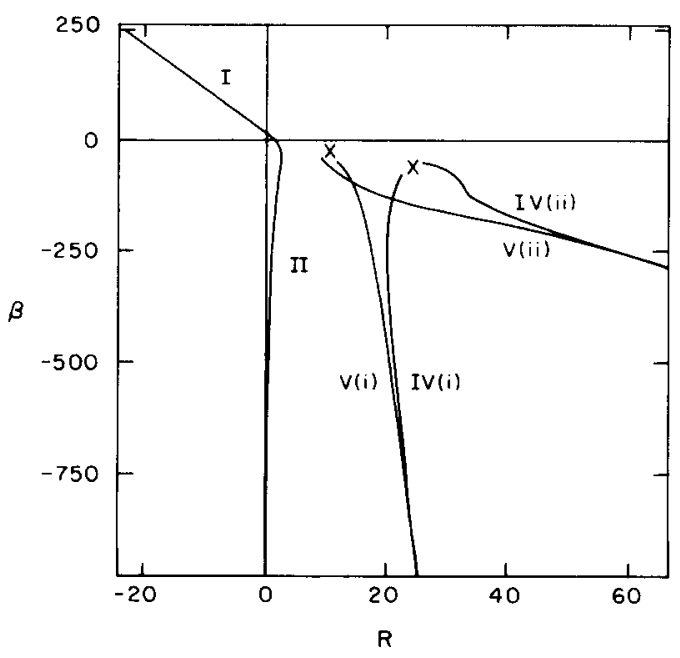

FIG. 7. The pressure coefficient $\beta$ of the porous tube similarity solutions as a function of the Reynolds numbers. The solution families are labeled groups I, II, IV(i), IV(ii), V(i), and V(ii) to be consistent with the classification scheme of Ref. 9. 
same state, whereas the groups IV(i) and V(i) do not, despite their similar $\beta$ vs $\mathrm{R}$ behavior. The structure of the accelerating tube problem is completely analogous.

Finally, Terrill and Thomas reported an additional family of solutions for negative $\mathbf{R}$ which they labeled group III. We were unable to reproduce these solutions (or to find them for the accelerating tube flow) and have determined that they are not solutions to the porous tube problem. Injection solutions for $\mathbf{R}$ negative, must have $v$ positive at the tube surface, i.e., $f(1)=1$. We were able to find the group III solutions reported by Terrill and Thomas with, however, $\mathbf{R}<0$ and $f(1)=-1$; thus, they are not legitimate solutions.

Proceeding as in Sec. II, the minimum magnitude eigenvalues for the porous tube groups I and II are shown in Fig. 8. For $R<0$, only negative eigenvalues were found, indicating the solutions are stable as was found for the two-dimensional case. For $\mathbf{R}>0$, both interpretations suggest that the group I solutions are stable, reaching a point of marginal stability when the shear stress at the wall vanishes at $\mathbf{R}=2.3$. The group II solutions have $\lambda<1$ and are unstable under either interpretation. The $\mathbf{R}<0$ stability results for the accelerating tube flow, which are not shown, resemble closely the porous tube results for negative Reynolds numbers. As is the case for the two-dimensional negative $R$ solutions, the two interpretations yield conflicting conclusions; the first implying instability for $-\infty<R<0$ and the second stability for $-\infty<R<0$. Group I accelerating tube stability results for $\mathbf{R}>0$ are shown in Fig. 9. They have the same form as the porous tube case, with $\lambda$ passing through unity at the limiting Reynolds number 10.25 . In the accelerating tube flow, the shear stress at the moving surface does not vanish at $R=10.25$, but the axial velocity profile does develop an inflection point, $f^{\prime \prime}(r)=0$ at $r$ near 1 , at this Reynolds number.

The minimum magnitude eigenvalues for the porous tube group $V$ solutions and the corresponding accelerating tube solutions are shown in Figs. 10 and 11. Brady and Acri$\operatorname{vos}^{13}$ labeled the accelerating tube families II and III, the

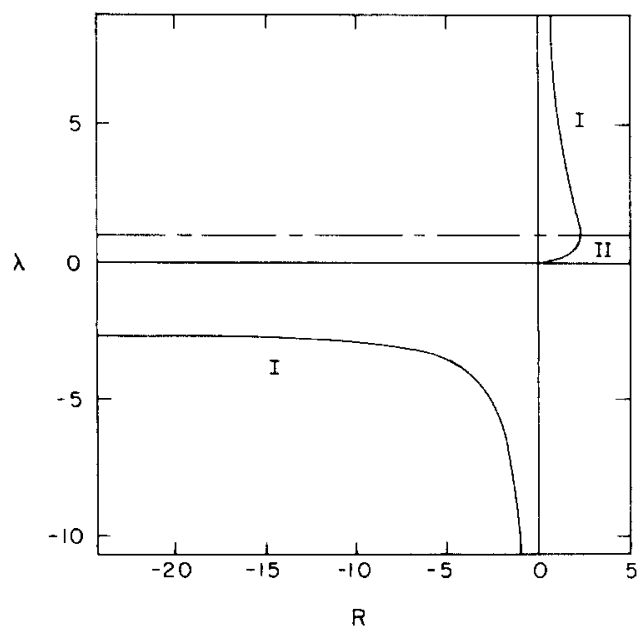

FIG. 8. The minimum magnitude eigenvalues $\lambda$ for the porous tube groups I and II. Here $\lambda=1$ at $R=2.3$ where the similarity solutions cease. Group II solutions are unstable.

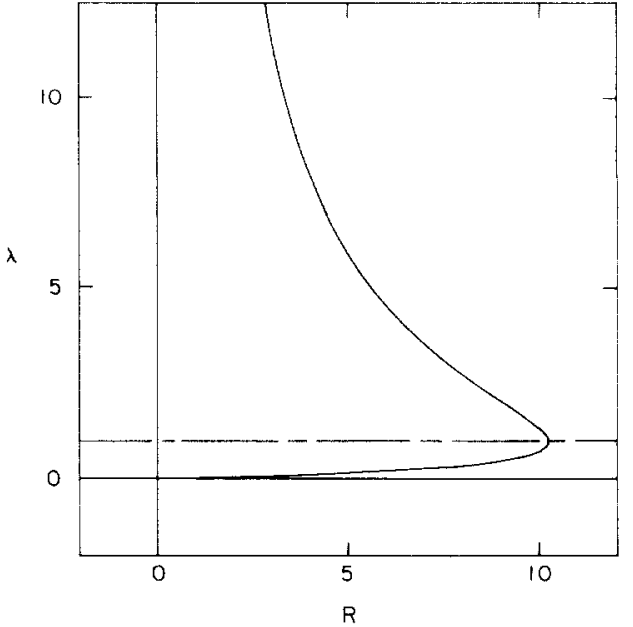

FIG. 9. The same as Fig. 8 for accelerating tube solutions. Here $\lambda=1$ at $R=10.25$ where the similarity solutions cease.

correspondence being V(ii) $\leftrightarrow$ II and V(i) $\leftrightarrow$ III. These eigenvalues should be compared with their two-dimensional counterparts in Figs. 5 and 6. As before, these solutions have both positive and negative $\lambda$ 's and are unstable under either interpretation.

One aspect of the tube solutions that differs from those in the channel is that the group V(ii) solutions are multivalued in the range $9.1 \leqslant R \leqslant 9.99$ while it was the group III solutions in two-dimensions that were multivalued. Thus in order that the small section of the $\mathrm{V}($ ii) solutions between $\lambda=1$ and the point $X$ (see arrow in Fig. 10) be unstable, the strongly negative $V(i)$ branch must cross over the point $X$ and pick up the $V$ (ii)'s before proceeding to $\lambda \rightarrow-\infty$. That is, the $V(i)$ branch in Fig. 10 must connect with the V(ii) branch, which is then multivalued as in the inset of Fig. 5. Attempts to complete this curve were hampered by the difficult numerical problem posed by very negative $\lambda$ 's, but we feel certain that the small section of group V(ii) solutions are indeed unstable. The same, of course, must be true for the accelerating tube solutions of Fig. 11.

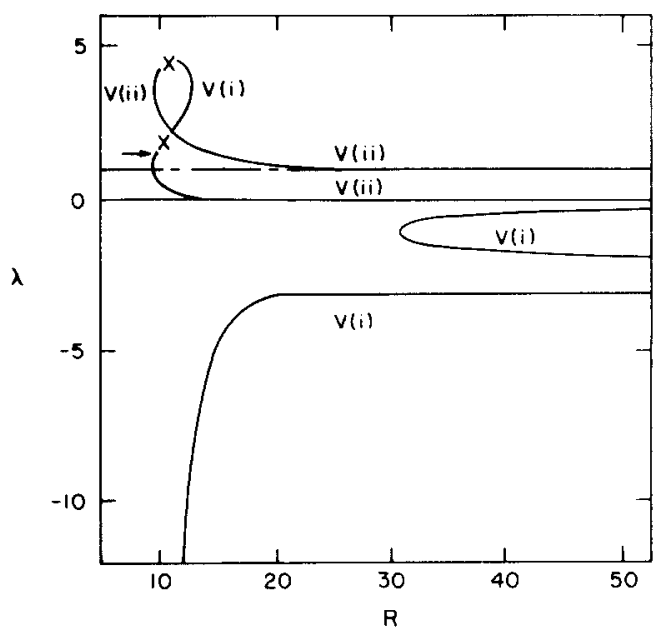

FIG. 10. The minimum magnitude eigenvalues for the porous tube group $\mathrm{V}$ (i) and $\mathrm{V}$ (ii) solutions. Note the resemblence to the two-dimensional counterpart in Fig. 5. The solutions are unstable. 


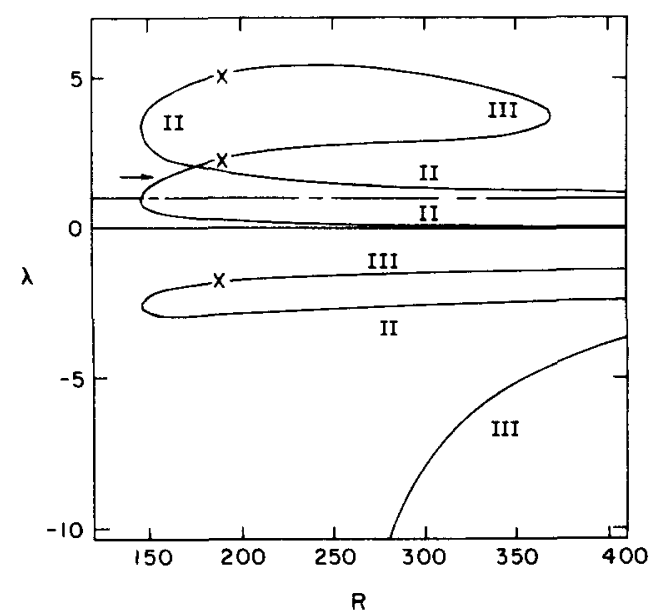

FIG. 11. Same as Fig. 10 for the accelerating tube solutions. The two-dimensional counterpart is Fig. 6.

In addition, unlike the channel solutions of Sec. II C, the minimum eigenvalues for the groups $\mathrm{V}$ (ii) and II tend to zero rather than unity asymptotically as $\mathrm{R} \rightarrow \infty$. This is significant because, with $\lambda \equiv 0$, the function $g^{\prime}(y)$ is not merely a pertubation, but an exact solution to the full equations of motion. That is, a similarity transformation of the form $u=-x\left[f^{\prime}(r) / r\right]-\left[g^{\prime}(r) / r\right]$, etc., satisfies the NavierStokes equations, with $g$ an eigensolution, i.e., $g$ satisfies a homogeneous equation and homogeneous boundary conditions and can have arbitrary amplitude. The boundary conditions on $g$ of $g(0)=g(1)=0$ ensure that the eigensolution produces zero net flux in the tube. Thus, asymptotically as $R \rightarrow \infty$, the $\lambda \rightarrow 0$ eigenvalues do not imply instability in the sense that the similarity solution breaks down, but rather imply the similarity form $u=-x f^{\prime}(r) / r$ is incomplete. The "full" similarity solution-linear in $x$ plus the eigensolution- is, however, marginally stable as $\mathbf{R} \rightarrow \infty$ due to the next higher eigenvalues approaching unity. Although strictly speaking the eigensolution $\lambda \equiv 0$ occurs only at infinite Reynolds number, at a Reynolds number of 20 in the porous tube flow and 350 in the accelerating tube flow the asymptotic state seems to have been reached. In his numerical study of the flow in a porous tube, $\mathrm{Brady}^{6}$ found that at Reynolds numbers in excess of 50 certain inlet velocity profiles gave flows which are superpositions of the eigensolution and the normal-linear in $x$-similarity solution as predicted by the present stability analysis.

Finally, in Fig. 12 we show the minimum magnitude eigenvalues $\lambda$ for the group IV porous tube solutions. The structure here is quite close to that of the group V in Fig. 10. As with the group $\mathrm{V}$ solutions, these solutions are unstable. We did not determine the stability of the corresponding accelerating tube solutions, but we expect results analogous to those shown in Fig. 12.

\section{CONCLUSIONS}

The spatial stability analysis of the porous wall and accelerating surface problems has led to some very interesting and important conclusions. Taken in total, the stability re-

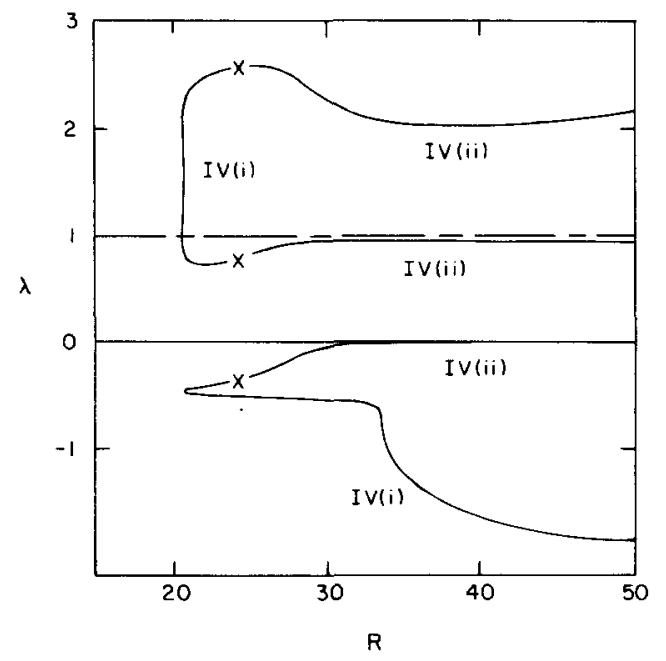

FIG. 12. The minimum magnitude eigenvalues $\lambda$ for the porous tube group IV solutions. These solutions are unstable.

sults indicate that the similarity solutions which have velocity profiles which are not monotonic in the cross-streamwise direction are unstable. The first interpretation we offered, which considers the entire flow as a whole, implies that only some of the monotonic solutions are stable, further limiting the range of stable solutions. The second interpretation implies that all monotonic solutions are stable, approaching marginal stability as $\mathbf{R} \rightarrow+\infty$ and remaining stable as $\mathbf{R} \rightarrow-\infty$. These conclusions, along with Serrin's proof of stability of the Falkner-Skan equation for $m \geqslant 0$, where the velocity profiles are also monotonic, suggest that it is the monotonicity of profiles that plays the key role. It is quite possible that these conclusions can be carried over into other classes of similarity solutions such as Jeffery-Hamel flow and the flow between two rotating disks. It is also possible that, based on monotonicity, it may be possible to prove analytically for arbitrary amplitude the spatial stability of boundary-layer-like solutions.

With this spatial stability analysis we were, however, unable to achieve completely our desired goal of predicting the critical Reynolds number for loss of validity of the similarity solutions as observed by Brady and Acrivos ${ }^{5}$ and Brady. ${ }^{6}$ For the axisymmetric problems, there is a critical Reynolds number which coincides precisely with the point where the similarity solutions cease to exist. This is really as one should expect, for it would be very surprising if $\lambda$ did not pass through 1 when solutions appear or disappear. For the porous channel problem, no critical Reynolds number was found. For the acceleration channel problem, a critical Reynolds number of 11 exists if the first interpretation is followed, but the second interpretation does not predict a critical Reynolds number.

The stability analysis has given us considerable insight into the nature of the similarity flows considered. Showing that the similarity solutions become marginally stable as $\mathbf{R} \rightarrow \infty$ helps to explain why one can find numerically, as in Ref. 6, solutions for the flow in a long but finite porous channel which are linear in $x$ as $x \rightarrow 0$ but not similarity solutions. These solutions are simply the similarity solution plus a perturbation with $\lambda=1$. The agreement between these full nu- 
merical solutions and the stability analysis can also be viewed as an indication of the utility and validity of the stability results. The temporal stability and spatial stability to a broader class of perturbations remain, of course, open questions.

\section{ACKNOWLEDGMENTS}

Discussions with Professor A. Acrivos are greatly appreciated, as is R. Reade's contribution to some of the numerical computations.

This work was partially supported by the DuPont Young Faculty Grant to J. F. B.

${ }^{1}$ H. Blasius, Z. Math. u. Phys. 56, 1 (1908)

${ }^{2}$ G. I. Barenblatt and Ya. B. Zel'dovich, Ann. Rev. Fluid Mech. 4, 285 (1972).

${ }^{3}$ J. B. Klemp and A. Acrivos, J. Fluid Mech. 76, 363 (1976).

${ }^{4}$ H. K. Moffatt and B. R. Duffy, J. Fluid Mech. 96, 299 (1980)

${ }^{5}$ J. F, Brady and A. Acrivos, J. Fluid Mech. 115, 427 (1982).
'J. F. Brady, Phys. Fluids 27, 1061 (1984).

${ }^{7}$ R. M. Terrill, Aeronaut. 15, 297 (1964).

${ }^{8}$ R. M. Terrill, Aeronaut. 16, 323 (1965)

${ }^{9}$ R. M. Terrill and P. W. Thomas, Appl. Sci. Res. 21, 37 (1969).

${ }^{10}$ W. A. Robinson, J. Eng. Math. 10, 23 (1976).

${ }^{11}$ F. M. Skalak and C-Y. Wang, Appl. Sci. Res. 33, 269 (1977).

${ }^{12}$ T. W. Secomb, J. Fluid Mech. 88, 273 (1978).

${ }^{13}$ J. F. Brady and A. Acrivos, J. Fluid Mech. 112, 127 (1981).

${ }^{14}$ J. Serrin, Proc. R. Soc. London Ser. A 299, 491 (1967).

${ }^{15}$ K. K. Chen and P. A. Libby, J. Fluid Mech. 33, 273 (1968).

${ }^{16} \mathrm{Chen}$ and Libby expressed the perturbation in the form $x^{-\lambda} \phi(\eta)$; in this case $\lambda>0$ would represent stability and $\lambda<0$ instability. To be consistent with our results, we have expressed their results in terms of a perturbation of the form $x^{\lambda} \phi(\eta)$.

${ }^{17}$ Solutions for complex $g, \lambda$, and $\beta$, were also sought; however, the only solutions found (by Newton's method and by searching in parameter space) were real.

${ }^{18}$ In obtaining Eq. (4) for $g$, we have neglected the term $\lambda(\lambda-1) x^{-2} g^{\prime}(y)$ which arises from $\partial^{2} u / \partial x^{2}$. Thus strictly speaking Eq. (4) does not apply at $x=0$. The stability question we are addressing is one in which the perturbation occurs on a length scale $L$ such that $h / L<1$, as was the case for the numerical solutions of Refs. 5 and 6 . Scaling $x$ with $L$ and $y$ with $h$ results in the above term having a coefficient $(h / L)^{2}$, which we can make arbitrarily small.

${ }^{19}$ B. C. Sakiadis, AIChE J. 7, 26 (1961).

${ }^{20}$ B. C. Sakiadis, AIChE J. 7, 221 (1961). 